\title{
Pharma Giant Expects Vaccines Lineup to Be Its Top Seller This
}

\section{Year}

Mangesh Panhale

Department of Pharmacy, India.

Corresponding Author: Mangesh Panhale, Department of Pharmacy, India.

Received date: November 06, 2021; Accepted date: December 18, 2021; Published date: January 06,2022

Citation Mangesh Panhale (2022) Pharma Giant Expects Vaccines Lineup to Be Its Top Seller This Year. J. Pharmaceutics and Pharmacology Research, 5(2); DOI:10.31579/2693-7247/038

Copyright: (C) 2022 Mangesh Panhale, This is an open access article distributed under the Creative Commons Attribution License, which permits unrestricted use, distribution, and reproduction in any medium, provided the original work is properly cited.

Summary
Coronavirus (COVID-19) was first seen in late December in Hubei province of Wuhan city in China. The highly
contagious disease, caused by a virus, severe acute respiratory syndrome coronavirus 2 (SARS-CoV-2), is transmitted
from humans to humans. After the first case in Wuhan, the disease rapidly spread to other parts of the globe.
Key words: covid-19; vaccine industry

\section{Overview}

Coronavirus (COVID-19) was first seen in late December in Hubei province of Wuhan city in China. The highly contagious disease, caused by a virus, severe acute respiratory syndrome coronavirus 2 (SARS-CoV2 ), is transmitted from humans to humans. After the first case in Wuhan, the disease rapidly spread to other parts of the globe. On 11th March 2020, the World Health Organization (WHO) made an assessment that COVID19 can be characterized as a pandemic. Thus, social-distancing became an important measure to stop the spread of this disease. Various countries across the world adopted nationwide lockdowns. This led to a completely new scenario for the world, where every business in each industry faced new challenges and witnessed new opportunities. Similarly, the vaccines industry, a vital part of the healthcare sector, was also hit in a deleterious manner. Before COVID-19, growth of the vaccines industry was expected to show a gradual increase, owing to various factors such as high investments in $R \& D$ and rise in awareness related to immunization. However, COVID-19 spread led to a sudden change in scenario, with unexpected changes in the vaccines industry. Major part of the vaccines industry was hit in a negative manner, whereas some areas grew, owing to rise in demand for vaccine against COVID-19.

The spread of the virus led to a major disruption in the starting phase of the lockdown where business related restrictions were strict, which, in turn, caused disturbances in supply chain as logistics were compromised in every part of the world initially. Other changes included incorporation of safety measures such as social distancing at work places and shortage of human resources.

Impact of COVID-19 on several industries in context with the vaccines industry

Vaccines are suspension of weakened or killed microorganisms or toxins or antibodies, which are administered to a healthy individual to prevent disease. These products are very vital in healthcare as these biological preparations are used to build immunity against deadly contagious diseases, which pose threat to human civilization. Children need immunity against different types of viral diseases in initial years where vaccines play a major role. A vaccine is administered by a qualified healthcare staff, which has sufficient knowledge about dosage and other aspects associated with the administration.

The COVID-19 outbreak impacted the vaccines segment in a negative manner in the initial month of the outbreak. Vaccination efforts that help to control infectious diseases were put on hold to concentrate on medical care for COVID-19. Medical staff was more inclined toward providing healthcare to COVID-19 patients to understand the new virus and stop its spread among healthcare individuals. This led to a scenario where needs related to vaccination were put on hold, owing to stringent social distancing measures. This majorly impacted availability of vaccines throughout the world during the outbreak. For instance, according to the National Foundation for Infectious Diseases, routine vaccination rates declined in the U.S. with demand plummeting as much as $95 \%$ for certain vaccines.

Similarly, according to a study conducted by the Centers for Disease Control and Prevention (CDC), fewer than half of infants aged 5 months and younger were up-to-date on recommended vaccinations in Michigan. In addition, according to a data from statewide immunization registry Florida SHOTS (State Health Online Tracking System), Miami-Dade County recorded an approximate $60 \%$ decline in children vaccines administered in April 2020, when compared to 2019. WHO also quoted that it is the first time in 28 years that the world could see a reduction in diphtheria-tetanus-pertussis (DTP3) coverage. A similar trend of decline in vaccination rates was seen in other parts of the world in the initial phase of the outbreak. Thus, this decline in demand for immunity providing vaccines negatively impacted the vaccines industry. 
As the airline industry collapsed during COVID-19 which had a profound impact on shipments of vaccines leading to a negative impact on the supply of vaccines. Profound decline in flights and closed airports at destination countries, owing to lockdown obstructed UNICEF's capability to ship vaccines as per country and supplier shipment plans. Although availability of commercial flights is improving with time, these improvements are limited. Some destinations are still difficult to access with limited flight and charter options. This disruption in supply chain has led to dire conditions in countries in various regions such as West and Central Africa, East and South Africa, and East Asia and South Asia. Stocks form major vaccines have reached critical levels in these regions, which include vaccines such as measles, Bacillus Calmette-Guérin (BCG), pentavalent (diphtheria, tetanus, pertussis (whooping cough), hepatitis $\mathrm{B}$, and haemophiles influenza type $\mathrm{b}$, human papillomavirus (HPV); hepatitis B, oral polio vaccine (OPV), rotavirus, and pneumococcal conjugate vaccine (PCV).
Moreover, with time stringent social distancing guidelines have eased and governments of several countries all over the globe have started taking measures to restore vaccination programs. In addition, importance of vaccination has led to restoration of vaccination programs in many parts of the world. World health organization has called on countries to make sure that immunization is maintained wherever possible. It is also ensuring that observation for vaccine preventable diseases remains undisrupted during the ongoing pandemic.

However, in the midst of a staggering impact on demand and supply of vaccines, COVID-19 outbreak also presented a huge opportunity to the vaccines industry. Measures to control the spread included vaccination against the virus, which can induce immunity in healthy individuals against COVID-19. This led to emergence of an urgent demand for vaccine against COVID-19. This demand is recognized by major key players across the globe and governments of nations worldwide.

\begin{tabular}{|l|c|c|c|c|c|c|c|c|c|}
\hline & 2019 & 2020 & 2021 & 2022 & 2023 & 2024 & 2025 & 2026 & 2027 \\
\hline Global Marketq & 32.5 & 34.5 & 36.7 & 39.1 & 41.74 & 44.6 & 47.5 & 50.7 & 54.1 \\
\hline
\end{tabular}

Table 1: Global Market, 2019-2027 (\$Billion)

\section{Expectations from the vaccination industry}

The vaccine industry is taking tremendous efforts to develop the first effective COVID-19 vaccine. Anticipation around the novel vaccine has become stronger as the virus is spreading at a rich pace. The sooner a vaccine is developed the more lives could be saved. Thus, the vaccine industry has a very dynamic role in the battle against COVID-19 infection.

Major players across the globe have started with research related to the vaccine and some products have reached late stages of clinical trials. In addition, governments, multilateral agencies, not-for-profit institutions, and the private sector are funding for research and development of COVID-19 vaccine. For instance, COVID-19 Therapeutics Accelerator (CTA) is a philanthropic collaboration. This collaboration supports efforts taken for R\&D to bring effective treatments against COVID-19 in market quickly and accessibly. Many vaccine giants have joined this program.

Companies such as Johnson \& Johnson (Janssen Pharmaceutical Companies), Cadila Healthcare Limited, GlaxoSmithKline plc, Merck Sharp \& Dohme, and Sinovac Biotech Ltd. are conducting research related to COVID-19 vaccines. Some vaccine products of these companies are present in the stage III clinical trials and some are in early stages of clinical trials. For instance, Johnson \& Johnson's candidate vaccine is currently being studied in phase III clinical trials. Other companies with their candidate vaccines in the stage III clinical trials include Sinovac Biotech Ltd. and Novavax, Inc.

There are approximately 48 candidate vaccines, which are currently being tested against COVID-19 in clinical trials on humans. Thus, strong pipeline vaccines are anticipated to hit the market in 2021 to change the COVID-19 scenario worldwide.

\section{Post COVID-19 Scenario}

As nations are emerging out of strict lockdowns, economy is expected to still need time to stabilize gradually. Players operating in the vaccine industry are anticipated to face new challenges related to different aspects. Decision making management of vaccine companies is anticipated to face challenges such as improvement of liquidity, management of working capital, better management of expenditures, and redefined contracts with suppliers.

Another scenario is anticipated to arise as governments of nations across the globe are becoming more particular about resuming immunization programs. Thus, this is expected to bring stability to the vaccine industry. Flights and land logistics are also anticipated to normalize, which may lead to steady rise in supply of vaccines post COVID-19.

Furthermore, the vaccine industry is expected to face a scenario related to constant demand for COVID-19 vaccine, once it is launched in the market. Certain factors are expected to become very important once the vaccines against COVID-19 are launched. For instance, vaccine manufacturers would have to adapt to a range of demand scenarios such as flexible manufacturing, collaboration with others to drive vaccine delivery, track the adoption, and monitoring of vaccines across various regions.

\section{Recent news in the vaccine industry during the pandemic}

1. Vaccine manufacturers around the world are actively participating and taking efforts toward the development of a vaccine effective against COVID-19.

2. On April 14, 2020, Sanofi S.A. and GlaxoSmithKline plc, entered into a collaboration to develop an adjuvanted vaccine for COVID19, using innovative technologies from both companies to help address the ongoing pandemic. Sanofi is expected to contribute its S-protein COVID-19 antigen, which is based on recombinant DNA technology and GSK would contribute its proven pandemic adjuvant technology.

3. On September 15, 2020, Novavax, Inc., announced an amendment to its existing agreement with Serum Institute of India Private Limited (SIIPL) under which SIIPL would manufacture the antigen component of NVX-CoV2373, Novavax' COVID-19 vaccine candidate.

4. On September 23, 2020, Sinovac Biotech Ltd., received National Medical Products Administration (NMPA) approval to study CoronaVac, inactivated COVID-19 vaccine candidate developed by company in clinical trial in adolescents and children. 
5. On October 23, 2020, Johnson \& Johnson, announced that it is preparing to resume recruitment in the pivotal Phase 3
ENSEMBLE trial of its investigational Janssen COVID-19 vaccine in the U.S. after a temporary pause. (c) $\oplus$

This work is licensed under Creative

Commons Attribution 4.0 License

\section{To Submit Your Article Click Here: $\quad$ Submit Manuscript}

DOI: $10.31579 / 2688-7517 / 038$
Ready to submit your research? Choose Auctores and benefit from:

fast, convenient online submission

$>$ rigorous peer review by experienced research in your field

$>$ rapid publication on acceptance

$>$ authors retain copyrights

$>$ unique DOI for all articles

$>$ immediate, unrestricted online access

At Auctores, research is always in progress.

Learn more https://auctoresonline.org/journals/pharmaceutics-andpharmacology-research 